\title{
New Alberta Coalition Aims to Tackle Cancer Through Healthy Public Policy
}

\author{
Janis Geary (University of Alberta) \\ News Reporter - HSI 2OIO-2OII
}

In the fall of 2010, the Alberta Policy Coalition for Cancer Prevention (APCCP) launched itself into the public arena. Funded by the Alberta Cancer Prevention Legacy Fund (Alberta Health Services) in 2009, APCCP brings together a diverse group of practitioners, policy makers, researchers and community organizations with a common goal: to develop and implement healthy public policies to reduce cancer risks.

To accomplish this, the Coalition aims to achieve three main objectives: (1) increasing capacity of policy makers in Alberta to use policy as a strategy for cancer and chronic disease prevention; (2) providing leadership in the development, implementation and evaluation of policy-related activities for cancer and chronic disease prevention; and (3) facilitating the collaboration of all stakeholders to work together to enhance public acceptance of policyrelated activities to address cancer risks.

The Alberta Cancer Board estimates that one in two Albertans will develop cancer in their lifetime. Across the country, cancer is the leading cause of premature death. Many cancers are preventable, and research has shown that more than $30 \%$ of cancers could be prevented by increasing physical activity, changing diets, avoiding tobacco use and alcohol misuse. Although these are all individual behaviours, changing the environment in which people make these choices can have a profound impact.

In the October 2010 media release, Coalition member Angeline Webb of the Canadian Cancer Society summed up the goals of APCCP, "Healthy public policy creates environments in which the healthy choice is the easy choice. Alberta has achieved some real success in the reduction of cancer risk factors. However, there are still key areas, where the implementation of healthy public policy can help prevent cancer".

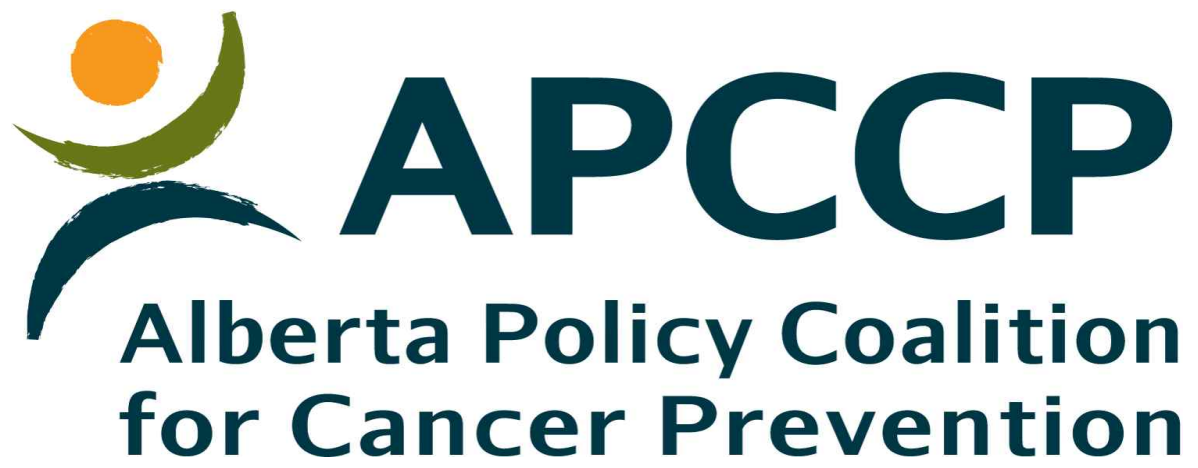

According to APCCP's Policy Analyst Shandy Reed, understanding where Alberta's strengths are across the broad field of cancer and chronic disease prevention, and where there are gaps, is key. One of the first tasks for the APCCP team was to complete an environmental scan of current policy activities in schools, communities and worksites in Alberta, Canada and internationally. This information was reviewed by the provincial advisory members and informed priority-setting for coalition action. In certain areas, groups such as Coalition for a Smoke-Free Alberta and the Alberta Center for Active Living have been achieving tremendous momentum in influencing policy changes. Accordingly, the APCCP's role is to support these efforts.

In other areas that may be less developed, the APCCP has identified a lead role for the coalition in furthering the set priorities. Reed works with Ken Kyle, a well-known advocacy consultant, to identify windows of opportunity for the coalition to use the evidence and take action. Examples where APCCP will be taking a lead include - banning marketing of unhealthy food and beverage products to children in schools, promoting active living in workplaces, encouraging taxation of energy-dense, nutrient-poor food and beverages, and promoting policies on urban design and zoning that promote active living and healthy eating. 
Two of the critical barriers to implementing public policies are acceptance of the public and willingness of decision makers. One of the first projects of APCCP research team was to better understand the knowledge, attitudes and beliefs of the Alberta public and decision makers regarding cancer prevention policies. 1,203 Albertans and 183 decision makers completed an APCCP survey, and the findings were shared in a media release on October 7, 2010. For the APCCP focus area on banning advertising and promotion of unhealthy foods and beverages to children under 16 , $82 \%$ of Albertans and $71 \%$ of decision makers were supportive.

Even in areas where there is a high level of acceptance for a particular policy intervention, Reed says achieving policy change is a long process. "Policy work is a long road which requires a sustained effort. It's often about small, incremental changes and successes. But when it all comes together, the positive impact for the population as a whole is well-worth the effort."

\section{"Policy work is a long road which requires a sustained effort. It's often about small, incremental changes and successes."}

Although Reed points out the amount of time and effort that is required to make policy change, she also acknowledges the impact that APCCP has already had in its short life-time. Over the last six months the APCCP has participated in a number of policy consultations, provided presentations to elected and senior government officials, surveyed school trustee candidates, and launched letter-writing campaigns and media releases in support of their priorities. Already leaders in developing healthy public policy, APCCP is facilitating collaborations that could lead to implementing policies that ultimately reduce the risk of chronic disease and cancer for Albertans. "The strength of the APCCP is in its membership. Our member organizations as well as our provincial and international advisory groups bring tremendous skill and expertise to the table. These resources paired with the APCCP's ability to stay nimble and respond to opportunities as they arise, are quickly making the APCCP a force to be reckoned with in Alberta."

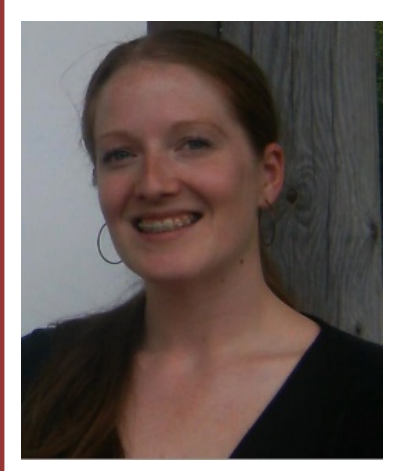

News Reporter Profile

Janis Geary is a 1st year PhD student in the School of Public Health at the University of Alberta. After completing her undergraduate degree in Microbiology at the University of Alberta, she moved to Edmonton to complete a Masters degree in Global Health. Since completing her masters she has been Project Manager for the Canadian North Helicobacter pylori Working Group. For her PhD, she is working on a project titled "Enhancing Trust and Communication in North-South Research Collaborations: A commons theoretical framework to equitable use and management of databases and biorepositories to support translational biomedical research". 